\title{
Equine Hoof Wall Tubule Density and Morphology
}

\author{
Lisa S. LANCASTER ${ }^{1)}$, Robert M. BOWKER ${ }^{1) *}$ and Whitney A. MAUER ${ }^{1)}$ \\ 1) Department of Pathobiology and Diagnostic Investigation and the Center for Comparative Epidemiology, College of Veterinary \\ Medicine, Michigan State University, East Lansing, Michigan 48824, U.S.A.
}

(Received 21 October 2012/Accepted 20 January 2013/Published online in J-STAGE 1 February 2013)

ABSTRACT. Density and morphologic features of hoof wall tubules in horses' hooves are described. The sample population consisted of hooves $(\mathrm{n}=12)$ from both live and deceased adult horses. Full wall thicknesses of the hoof wall were prepared histologically from sites at the toe, medial quarter and lateral quarter. In defined areas, tubules were counted, and density was calculated across the three histologically defined areas of the stratum externum, stratum medium and stratum internum along with the descriptive morphologic features of the tubules. Morphologically, distinct zones were demarcated by varying tubular cortical and medullary characteristics. Tubule density showed a radially increasing pattern across the wall thickness. Significant regional differences in density were found in the toe, lateral and medial walls. These findings represent a first study of regional differences of tubular densities. Hoof horn clippings from live feet may have potential diagnostic and/or prognostic value.

KEY WORDS: anatomy, equine, hoof, tubule.

doi: 10.1292/jvms.12-0399; J. Vet. Med. Sci. 75(6): 773-778, 2013

Equine hoof wall is composed of modified epidermal tissue arranged in both laminar and tubular architecture. Research directed towards understanding the functional architecture of the laminae suggests that the structure may be altered with environmental stresses, even without overt disease [9-12, 20]. Hoof wall tubules along the dorsal wall have also been examined and are considered to serve a primarily mechanical role $[1,5,7,8,14,16,19]$, although the details of mechanical function remain unclear at this time. Early work suggested tubules functioned to resist compressive load [13]. Later work suggested that tubules provide reinforcement in a composite structure [1]. This potentially reinforcing property of tubules appears to offer fracture and crack-resistance $[1,8]$, with intertubular horn providing more stiffness and fracture toughness than tubular horn $[1,11]$. Reilly $[16,17]$ identified a tubule density gradient across the hoof wall, proposing that a zonal architecture offered smooth energy transfer as well as crack-stopping properties.

Previous works have observed that the primary epidermal laminae (PEL) densities vary in different horse populations and this variation may relate to stresses along the hoof wall $[2,6,10,21]$. However, studies examining equine hoof wall tubules and/or tubular density have not yet shown, if or how tubular architecture may vary or respond to environmental forces in ways similar to laminae. Only Reilly [16, 17], to the best of our knowledge, has defined tubular density relationships objectively. However, there are no reports of tubule density or morphology at the lateral and medial hoof wall locations.

\footnotetext{
*Correspondence to: Bowker, R. M., Department of Pathobiology and Diagnostic Investigation, College of Veterinary Medicine, Michigan State University, East Lansing, Michigan 48824, U.S.A. e-mail: Bowker@cvm.msu.edu

(C)2013 The Japanese Society of Veterinary Science
}

Our interest in the present study was to collect novel data that would allow us to generate hypotheses for future research on three topics related to tubule characteristics in different horse populations. First, we wanted to investigate whether the hoof wall tubules exhibited variation in density or structure in medial and lateral hoof wall locations or if tubules in these locations differed from what was previously documented at the toe wall. Second, we wanted to document these tubule characteristics in different horse populations of known age and occupation to identify potential trends, such as environmental influences that may determine their distribution characteristics. Third, we chose to include hoof horn samples from live horses, as this method may be used to investigate diagnostic or prognostic potential.

\section{MATERIALS AND METHODS}

Sample collection: Hoof wall samples were obtained from 12 horses (the left front foot from each horse) including live and cadaveric feet: 4 racing Quarter Horse $(\mathrm{QH})$ and 3 feral horse cadavers and 5 live pleasure horses (4 QH and 1 Arabian). The $\mathrm{QH}$ racehorses were euthanized after catastrophic injury (unrelated to the feet). The feral feet were collected during a Bureau of Land Management roundup in the western United States. Race and feral horses were all 3 years old at the time of death. The live horses ranged in age from 4 to 28 years old.

Cadaver feet were disarticulated at the proximal interphalangeal joint, frozen and then cut with a band saw in cross-section parallel to the ground surface. Samples from live pleasure horses were obtained from the bearing surface of hooves during routine farrier trimming.

Sample preparation: From the cadaver feet, samples cut in 5-6 mm-thick slices were collected at the toe, medial quarter and lateral quarter from the left front foot of each horse (Fig. 1). Rectangular sections $1 \times 2 \mathrm{~cm}$ through the hoof 


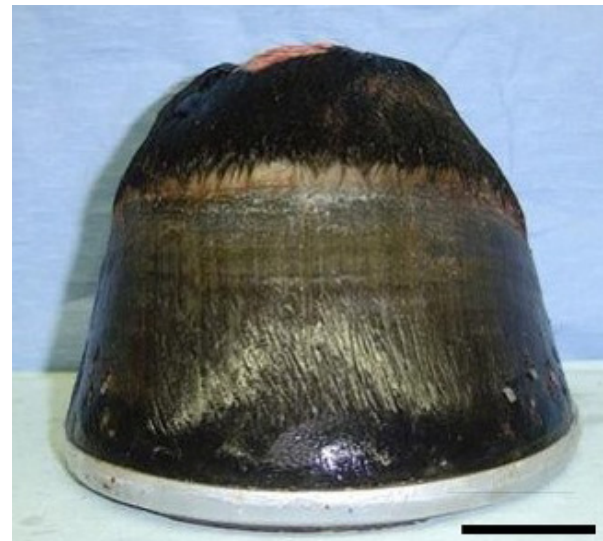

Fig. 1. Photograph of 3 years old race horse foot showing representative conformation of sample feet, dorsal view; bar $=3.60 \mathrm{~cm}$.

wall to the corium or distal phalanx were removed, placed in buffered formalin (pH 7.4 to 7.8) and processed for paraffin embedding. From the live horses, the farrier trimmings were collected, cut into $1 \times 2 \mathrm{~cm}$ rectangles from toe and quarter areas, placed in formalin and processed similarly. All sections were processed for HE and Masson trichrome histochemistry.

Data collection: Histological slides were digitized with a calibrated marker using Bioquant MEQ IV software. Digital photographs were combined in Photoshop (Adobe Photoshop CS3 Extended, 2007, San Jose, CA, U.S.A.) to recreate the original images. A two by two millimeter grid was overlaid on photographs, and tubules were counted at adjacent zones across the plane from the inner hoof wall laminae (stratum internum) to the outer wall of the stratum medium and stratum externum. This plane defined the tubular orientation from the center of the internal wall (axially) towards the abaxial surface at toe, medial quarter and lateral quarter. Tubule density was calculated following Reilly's method of density measurements [16] as the number of tubules per square $\mathrm{mm}$. Tubule density was initially calculated in $10 \%$ increments across the depth of hoof wall with $100 \%$ at the innermost edge, (dermal edge of the stratum internum) and $0 \%$ being the outermost edge (stratum externum, also called periople). Data were then combined into 4 zones (each zone equaled approximately $25 \%$ depth of hoof wall). On photographs, two rows of blocks $(1 \times 1 \mathrm{~mm})$ were counted and then averaged to get tubule density per square $\mathrm{mm}$ by percentage of hoof wall depth. A tubule was counted, if its full medulla was within the bounds of a block. The first row of blocks was counted along the abaxial edge of laminar junction. Tubules were counted to the outer-most edge of hoof wall, including the stratum externum (periople). If the last block was not a complete $\mathrm{mm}$, the fraction was estimated and tubule density per square $\mathrm{mm}$ for that zone was interpolated.

Statistical analysis: Analyses were conducted on the tubule density data using commercial statistical software. Domestic feet from race and pleasure horses were combined $(n=9)$. Normality of the data was established using ShapiroWilks test, and data were considered statistically significant if $P<0.05$ was achieved. Two-tailed $t$-tests were used to compare toe to quarters, and to compare quarter to quarter within each foot.

\section{RESULTS}

Circumferential zonal pattern: In all feet sampled, there was a radial pattern of increased tubule density across the axial to abaxial plane. This was consistent around the entire circumference of the foot. Tubule density was lower at the axial (dermal) edge than the abaxial (hoof wall) edge. Combining all samples at the distal-most sample level (which was below the distal margin of P3), the radial increase in tubular density across the axial-abaxial plane was not a smooth curve. From the axial (dermal) edge of the laminar tubule junction moving abaxially, there was a slight drop in tubule density within zone 1, at approximately $90 \%$ of hoof wall depth. For each remaining zone, there was an increase in tubular density. Although the general axial-abaxial increase in tubule density in zones $2-4$ was consistent across all samples, there were variations in the curve shape between toe, medial and lateral quarters. There were also differences in the curve shape between feral and domestic specimens. Changes in distribution of tubules are shown graphically (Fig. 2A and 2B).

Regional tubule density: The radial pattern noted above was consistent from the axial to abaxial edge at any point on the hoof's perimeter. But, the average tubule density varied across different sample sites in the toe and quarters (Table 1). All feet had higher tubule density in the toe than in the lateral quarter $(P<0.05)$. The quarter with higher tubule density was on the medial side of the foot. The difference in tubular density between medial and lateral quarters was statistically significant as was the difference between toe and lateral quarter $(P<0.05)$. The difference between toe and medial quarter was not statistically significant. Comparing the radial pattern from medial to lateral, an increase in zone 4 was most pronounced on the medial quarter and least pronounced on the lateral quarter. There were tubular density differences around the circumference of the hoof wall with more tubules per square $\mathrm{mm}$ in zone 4 on the medial quarter compared to the lateral quarter. Feral feet showed an overall lower tubule density at the toe wall compared to domestic feet, but there was a higher density in the outermost zone than in domestic feet. Medial and lateral samples were not available from feral feet.

Morphology: Three subpopulations of morphologically distinct tubules were identified. Morphological differences were noted between tubules in zones 1, 2-3 and 4. In zone 1 , adjacent to the abaxial extent of the laminar junction, tiny horn tubules closest to the laminae were oriented in line with the dermal tips and appear to be "budding off" from secondary epidermal laminae between two neighboring primary epidermal bases. Approximately $2 \mathrm{~mm}$ abaxially from the dermal edge, there was a zone approximately $2 \mathrm{~mm}$ wide where the tubules were the largest with the most well defined 
A

\section{Medial and Lateral Wall Tubule Density}

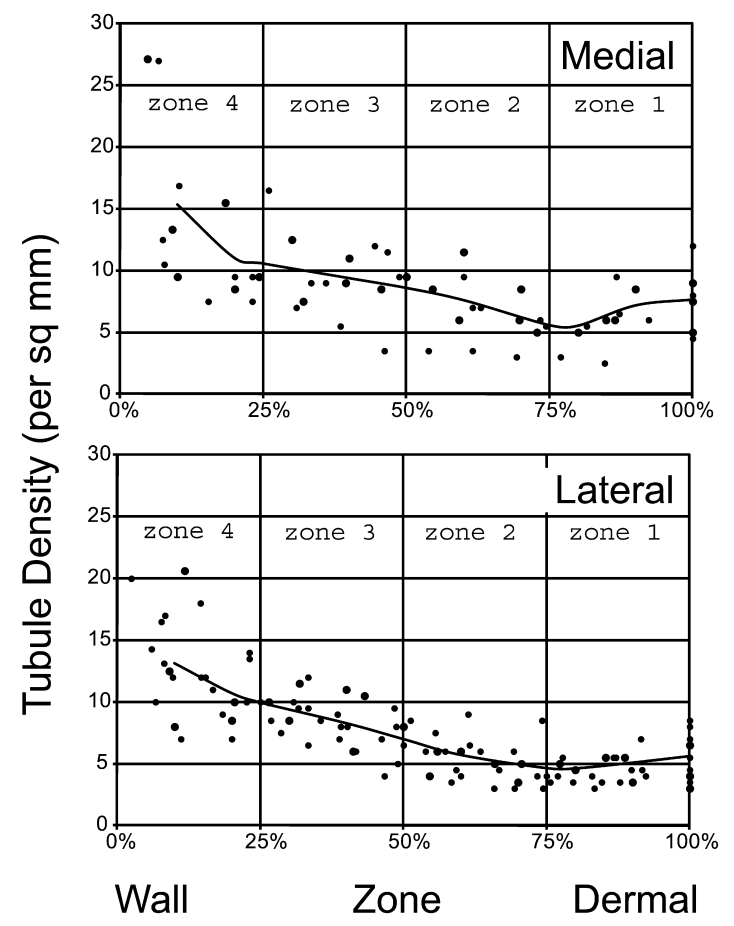

B Toe Wall Tubule Density

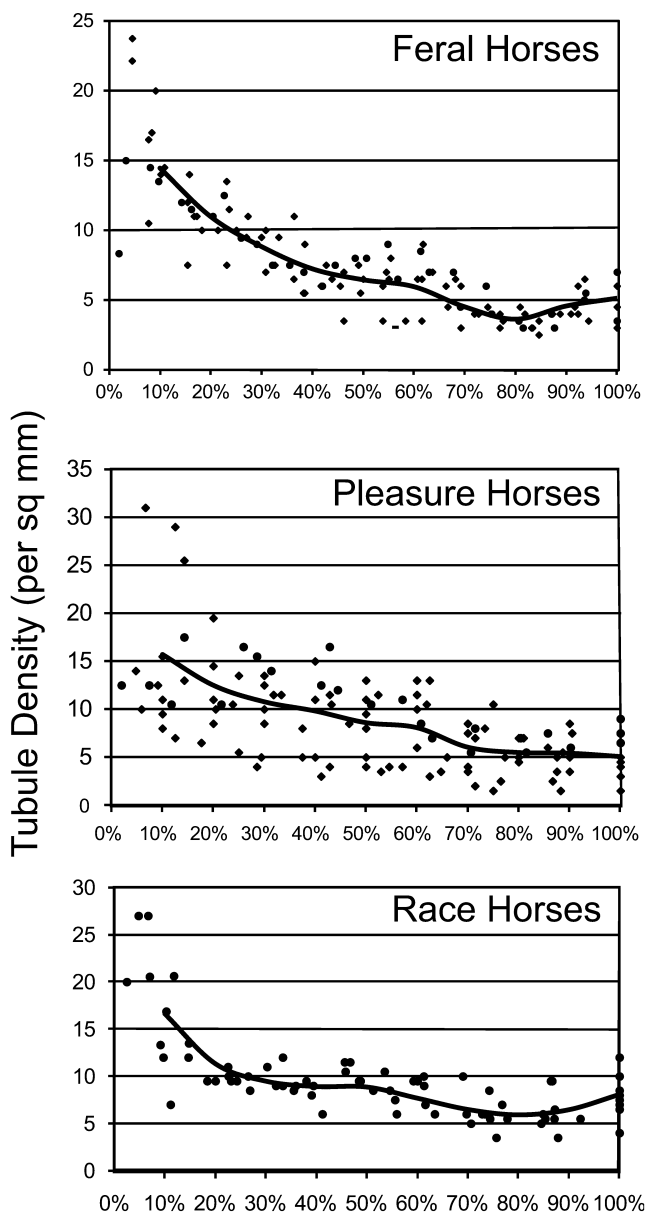

Fig. 2. A: Graphs of tubule density of medial and lateral walls, data combined from pleasure and race horses $(\mathrm{n}=9)$. The higher density on the medial compared to the lateral was statistically significant $(P<0.05)$. B: Graphs of tubule density at the toe wall of feral horses $(\mathrm{n}=3)$, pleasure horses $(n=5)$ and racehorse $(n=4)$.

Table 1. Tubule density per square millimeter-regional differences in domestic and feral horse feet

\begin{tabular}{lccc}
\hline & Toe & Medial $^{\text {a, c) }}$ & Lateral $^{\text {b) }}$ \\
\hline Domestic (n=9) & $\begin{array}{c}\text { Range 6.5-14.3 } \\
\text { Average 10.4 }\end{array}$ & $\begin{array}{c}\text { Range 6.0-16.3 } \\
\text { Average 11.2 }\end{array}$ & $\begin{array}{c}\text { Range 5.1-12.2 } \\
\text { Average 8.7 }\end{array}$ \\
\hline Feral (n=3) & $\begin{array}{c}\text { Range 4.8-15.1 } \\
\text { Average 9.9 }\end{array}$ & N/A & N/A \\
\hline
\end{tabular}

a) Difference between medial and lateral $P=0.00048$. b) Difference between toe and lateral $P=0.000025$. c) Difference between toe and medial not statistically significant $P=0.22$.

cortical zone and largest medullary cavities (approximately zones 2-3). Continuing abaxially, the tubule cortices became less well-defined, but they had a fairly uniform appearance. In the outermost millimeter of hoof wall, tubules were similar in size and density to the rest of the outer hoof wall, but consistently showed uptake of stain similar to that of the inner hoof wall.

\section{DISCUSSION}

Circumferential zonal pattern of tubule density: Mean tubule density (MTD) data in the present study are consistent with previous findings of a radial increase of tubular density from inner to outer edge of the hoof at the toe $[11,16,17$, 19]. The present study reported for the first time data on tu- 
bule density in locations other than the toe. The medial and lateral locations show the same radial pattern, but there was a significant difference in tubule density between the medial and lateral sides, and between the toe and the lateral quarter.

Based on a previous study on regional variation in laminar density around the hoof wall circumference [10], we anticipated that tubule density patterns may be related to the laminar density patterns. We found that hoof wall tubule density patterns were generally similar to laminar density patterns, but did not appear to correspond uniformly. Like epidermal laminar density, tubule density was highest in the toe region of the hoof compared to the lateral quarter. In contrast to epidermal laminar density, tubule density was higher in the medial quarter than lateral quarter in the stratum medium. The wall tubule density at the toe was more similar to the medial quarter than to the lateral quarter. The greater tubular density may be directly related to a greater wall thickness with the additional tubules being formed from keratinocyte movements from the adjacent laminae (unpublished observations). The wall at the toe and the medial side of the hoof are usually the thicker regions of the hoof wall and are often subjected to greater loading forces depending upon hoof's conformation [4, 15]. Nickel [13] suggested that more rapid wear induced the horn tubules to become more crowded. These thickened wall areas may have greater tensile forces within the stratum medium of the wall, requiring reinforcement from additional tubules. Hoof wall tubules may respond differently than the laminae do to similar loads. It is also possible that tubular horn responds in a similar fashion to the laminae, but that the physical forces on the tubular horn are different than the loads occurring in the laminar junction.

Previous works [17] on MTD across the stratum medium indicate a large range from 5 to 48 tubules per $\mathrm{mm}$ with a mean of 16 tubules per mm squared. Wall sampling locations vary, or were not described sufficiently, among the studies, which may explain the wide range differences in the findings. Reilly et al. $[16,17]$ sampled at a specific site - the midpoint between the coronet and ground surface - whereas our samples were consistently obtained in the distal third of the hoof wall. The present study of MTD at the toe was less (average of 10.4 tubules/mm for domestic horses and 9.9 for feral horses) than that the study of Reilly et al., but close to those observations of others. While any possible differences between our studies may be a result of technical matters during measurements — discussed by Reilly [17] — differences in environmental factors could also contribute to the variations in MTD in the different hooves examined.

We chose to examine different groups of horses of known age and occupation as these data may begin to reveal environmental influences explaining differences in tubule density findings from disparate studies. Previous work examined tubule density in 8 ponies ranging from 2-14 years of age (use was not reported) [16], and in 8 slaughterhouse horses of unknown age or use [17]. Reilly proposed three possible explanations for significantly lower tubule density in the outer 2 zones and significantly higher density at the innermost zone of horses compared to ponies [17]. There may be a functional difference between horse and pony horn, there may be methodological differences in the way the two studies measured tubule density, or there may be age-related differences in tubule density [17]. The age-related factor may be important, but from the previous studies, it is difficult to assess because the ponies had a wide age range and the horses ages were unknown, although it is possible there was a bias towards older horses as they were random slaughterhouse samples [17]. It may be the case that slaughterhouse samples are biased towards older horses, but with a small sample of 8 horses, it is not possible to draw this conclusion. Although there are no reports of age related tubule density in horses, Reilly mentioned that studies of cloven hooved animals have suggested that tubule density decreases with age, and suggested that may be the case in horses too [17].

If there is a correlation between older horses and lower tubule density, we would expect our pleasure horses, age range 4-28, to have lower tubule density than our 3 years old race and feral horses. But, our data show the opposite of this trend. Data shown in the graphs from each group (Fig. 2B) reveal that feral and racehorses have lower overall tubule density and a "tighter" scatter plot than that of the pleasure horses. It is possible older horses have higher density and that Reilly's samples from unknown ages [17] came from younger horses, which would bring that data in line with ours. We do not have a large enough sample to firmly establish any correlation but knowing the age and use of our samples permits us to develop hypotheses for future research. Other influences on tubule density that could explain our data differences between the race, feral and pleasure horses may include trimming or shoeing practices, horse occupation or ground surfaces of the living environment. Although this did not apply to any of our samples, disease conditions affecting the hoof wall, such as laminitis may also influence tubule characteristics. The method of preparing histology slides from horn clippings of live horses will be useful for designing studies to test any of these potential environmental influences.

Regional tubule density variation: The present study provided new findings of tubular densities at the quarters of the foot. Although the radial increase in tubular density was identified at every location studied, the tubule density was not the same in the medial and lateral quarters (Fig. 2A). The tubular density was significantly higher in the medial quarters compared to the lateral quarters, but not significantly different from the toe region of the hoof wall. These differences in the tubular densities on the medial and lateral sides of the hoof suggest the possibility that environmental loading may be a factor in determining the structure and/ or distributions of the tubules within the hoof wall.

Our findings of different MTD in the wall quarters may provide further insights into the tubular functions and possible adaptive changes in relation to physical forces on the wall. Tubules have long been thought to be the primary support strength of the hoof wall [14]. Leach [11] hypothesized that when the wall was subjected to vertical compression forces, the tubules may augment the rigidity of the intertubular horn. The distribution of the loads and the greater MTD on the medial wall (more upright) are consistent with 
this notion that tubules are supportive structures. However, during hoof loading superficial wall measurements of the forces do not consistently align with tubular orientation [21]. Alternatively, greater tensile and strain forces may be occurring internally, producing a stratification of physical forces within the stratum medium. Such high forces may result in a shifting or movement of tubules and intertubular horn within the different regions of the hoof in order to reduce or minimize areas of peak stresses. Little is known of tubular orientation and physical force alignment within the deeper internal regions of the stratum medium.

As noted above, using horn samples from live horses would permit testing of mediolateral weightbearing correlations with tubule densities. Horses with different conformations, such as marked differences in wall angle medial to lateral, could be compared to horses with more symmetrical feet. Or, horses living on different surfaces or doing different jobs could be compared. Our samples all came from feet that were nearly symmetrical medial to lateral (Fig. 1). Given the lack of obvious difference in wall angle, we expected tubule density to be similar from medial to lateral. If tubule density is related to hoof wall loading patterns, the findings of significant differences between medial and lateral in grossly symmetrical appearing feet suggest that with more obvious distortion, which is quite common across the horse population (personal observations), we might find larger differences between medial and lateral tubule density in a larger sample. We do not yet know if there is an optimal density difference between medial and lateral sides of the hoof. Even if such data could be established, we do not know whether there would be any husbandry practices that would encourage hooves to generate this beneficial tubule density ratio.

Morphology: The innermost region adjacent to the stratum internum where the smallest tubules are located was not apparently included in the densitometric measurements of previous studies. While it is unclear if this innermost region, often call the capped horn, is part of the stratum medium or is unique in distribution along the interface of the two inner wall layers, plentiful tubule numbers along the inner wall do suggest that this region is active in tubular production from the PEL $[2,3,18]$. This inner most zone of tubules appears to be a transitional zone between inner wall and laminar interface. In this transition zone, stronger physical forces may be impinging upon the tissues, and the tubules may reduce the stresses being transferred to the cortical surface of the distal phalanx. Perhaps, the outer/superficial layers of these larger tubules may contribute to the intertubular horn matrix (unpublished observations).

Some research [8] has suggested that the smaller and more densely packed tubules in the outer wall may prevent fractures. The intertubular horn may have equal, if not more significance in distributing the physical forces within the wall $[1,11]$. The intertubular horn may shift or move within the stratum medium with inner wall strains and stresses permitting a reduction of high peaks of physical force from occurring in any one wall region. At different proximodistal regions of the hoof wall, tubules may bend and/or shift according to the shear forces within the stratum medium. The present study is the first documentation of tubule density patterns on the medial and lateral sides of the hoof. In this study, we described and quantified histologic features of the tubular horn in different circumferential locations of the hoof. Our data suggested that hoof horn tubule density may be influenced by environmental factors including age, occupation or farrier practices. Our method of using hoof horn from live horses as well as cadaver feet from horses with known age and occupation will permit a future study of larger sample populations to test hypotheses about factors associated with hoof horn tubule density and architecture.

ACKNOWLEDGMENTS. This study was supported in part by the American Quarter Horse Association and the United States Equestrian Federation.

\section{REFERENCES}

1. Bertram, J. E. and Gosline, J. M. 1987. Functional design of horse hoof keratin: the modulation of mechanical properties through hydration effects. J. Exp. Biol. 130: 121-136. [Medline]

2. Bowker, R. M. 2003. The growth and adaptive capabilities of the hoof wall and sole: functional changes in response to stress. $\mathrm{Am}$. Assoc. Eq. Pract. 49: 146-168.

3. Budras, K. D., Hullinger, R. L. and Sack, W. O. 1989. Light and electron microscopy of keratinization in the laminar epidermis of the equine hoof with reference to laminitis. Am. J. Vet. Res. 50: 1150-1160. [Medline]

4. Colahan, P., Leach, D. and Muir, G. 1991. Center of pressure location of the hoof with and without hoof wedges. Equine Exerc. Physiol. 3: 113-119.

5. Douglas, J. E., Biddick, T. L., Thomason, J. J. and Jofriet, J. C. 1998. Stress/strain behaviour of the equine laminar junction. $J$. Exp. Biol. 201: 2287-2297. [Medline]

6. Faramarzi, B. 2011. Morphological spectrum of primary epidermal laminae in the forehoof of Thoroughbred horses. Equine Vet. J. 43: 732-736. [Medline] [CrossRef]

7. Kainer, R. A. 2002. Functional anatomy of equine locomotor organs. pp. 1-72. In: Adam's Lameness in Horses, 5th ed. (Stashak, T. S. ed.), Lippincott Williams \& Wilkins, Philadelphia.

8. Kasapi, M. A. and Gosline, J. M. 1998. Exploring the possible functions of equine hoof wall tubules. Equine Vet. J. Suppl. (26): 10-14. [Medline]

9. Kawasako, K., Higashi, T., Nakaji, Y., Komine, M., Hirayama, K., Matsuda, K., Okamoto, M., Hashimoto, H., Tagami, M., Tsunoda, N. and Taniyama, H. 2009. Histologic evaluation of the diversity of epidermal laminae in hooves of horses without clinical signs of laminitis. Am. J. Vet. Res. 70: 186-193. [Medline] [CrossRef]

10. Lancaster, L. S., Bowker, R. M. and Mauer, W. A. 2007. Density and morphologic features of primary epidermal laminae in the feet of three-year-old racing Quarter Horses. Am. J. Vet. Res. 68: 11-19. [Medline] [CrossRef]

11. Leach, D. H. 1980. The Structure and Function of Equine Hoof Wall, PhD Thesis, University of Saskatchewan, Saskatchewan.

12. Linford, R. L., O’Brien, T. R. and Trout, D. R. 1993. Qualitative and morphometric radiographic findings in the distal phalanx and digital soft tissues of sound Thoroughbred racehorses. Am. J. Vet. Res. 54: 38-51. [Medline]

13. Nickel, R. 1938. Uber den Bau der Hufrohrchen und seine Bedeutung fur den Mechnismus Pferdehufes. Morphologisches 
Jahrb. 82: 119-160.

14. Pollitt, C. C. 1992. Clinical anatomy and physiology of the normal equine foot. Equine Vet. Educ. 4: 219-224. [CrossRef]

15. Ratzlaff, M. H., Wilson, P. D., Hyde, M. L., Balch, O. K. and Grant, B. D. 1993. Relationships between locomotor forces, hoof position and joint motion during the support phase of the stride of galloping horses. Acta Anat. (Basel) 146: 200-204. [Medline] [CrossRef]

16. Reilly, J. D., Cottrell, D. F., Martin, R. J. and Cuddeford, D. 1996. Tubule density in equine hoof horn. Biomimetics 4: 23-36.

17. Reilly, J. D., Collins, S. N., Cope, B. C., Hopegood, L. and Latham, R. J. 1998. Tubule density of the stratum medium of horse hoof. Equine Vet. J. Suppl. (26): 4-9. [Medline]

18. Sarratt, S. M. and Hood, D. M. 2005. Evaluation of architectural changes along the proximal to distal regions of the dorsal laminar interface in the equine hoof. Am. J. Vet. Res. 66: 277-283. [Medline] [CrossRef]

19. Schummer, A., Wilkens, H., Vollmerhaus, B. and Habermehl, K. H. 1981. The Circulatory System, the Skin and the Cutaneous Organs of Domestic Animals, Verlag Paul Parey, Berlin.

20. Stump, J. E. 1967. Anatomy of the normal equine foot, including microscopic features of the laminar region. J. Am. Vet. Med. Assoc. 151: 1588-1598. [Medline]

21. Thomason, J. J., McClinchey, H. L. and Jofriet, J. C. 2002. Analysis of strain and stress in the equine hoof capsule using finite element methods: comparison with principal strains recorded in vivo. Equine Vet. J. 34: 719-725. [Medline] [CrossRef] 